\title{
Directional Dyadic Wavelet Transforms: Design and Algorithms
}

\author{
Pierre Vandergheynst, Member, IEEE, and Jean-François Gobbers
}

\begin{abstract}
We propose a simple and efficient technique for designing translation invariant dyadic wavelet transforms (DWTs) in two dimensions. Our technique relies on an extension of the work of Duval-Destin et al. [1], [2] where dyadic decompositions are constructed starting from the continuous wavelet transfrom. The main advantage of this framework is that it allows for a lot of freedom in designing two-dimensional (2-D) dyadic wavelets. We use this property to construct directional wavelets, whose orientation filtering capabilities are very important in image processing. We address the efficient implementation of these decompositions by constructing approximate QMFs through an $L^{2}$ optimization. We also propose and study an efficient implementation in the Fourier domain for dealing with large filters.
\end{abstract}

Index Terms-Dyadic wavelet transform (DWT), QMF filters.

\section{INTRODUCTION}

W AVELETS are a well-established tool in the field of image analysis. In particular wavelet orthogonal and biorthogonal bases have been succesfully used in many applications. It has already been stressed that the lack of translation invariance of these representations can be a severe drawback for tasks like pattern recognition or texture analysis. The dyadic wavelet transform (DWT) pioneered by Mallat and Zhong [3] was precisely introduced to cope with this and has proved very useful when analysis of multiscale features is important [4], [5]. On the other hand, it has also been shown that the use of specific orientation sensitive filters like Gabor filters or directional wavelets is highly suitable when one faces multiscale oriented tokens in images [6], [7]. The main goal of this paper is the wedding of the powerful and simple algorithmic structure of dyadic wavelet frames together with rather mild design constraints. This will allow us to tailor two-dimensional (2-D) wavelets to obtain directional selectivity or suitable frequency localization properties without much effort.

Let $f$ be a square integrable 2-D signal, $f \in L^{2}\left(\mathbb{R}^{2}, d^{2} \vec{x}\right)$ and $\hat{f}$ be its 2-D Fourier transform defined by

$$
\hat{f}(\vec{\omega})=\int_{\mathbb{R}^{2}} d^{2} \vec{x} f(\vec{x}) e^{-i \vec{\omega} \cdot \vec{x}}
$$

Manuscript received November 10, 1999; revised December 13, 2001. This work was supported by FRIA, Belgium. The associate editor coordinating the review of this manuscript and approving it for publication was Dr. Eric L. Miller.

P. Vandergheynst is with the Signal Processing Laboratory, Swiss Federal Institute of Technology (EPFL), CH-1015 Lausanne, Switzerland (e-mail: Pierre.Vandergheynst@epfl.ch).

J.-F. Gobbers is with the Institut de Physique Théorique, Université catholique de Louvain (UCL) 2, B-1348 Louvain-la-neuve, Belgium and also with the Remote Sensing Laboratory, UCL, Louvain-la-neuve, Belgium (e-mail: gobbers@fyma.ucl.ac.be; gobbers@tele.ucl.ac.be).

Publisher Item Identifier S 1057-7149(02)03977-5.
Throughout this paper, the $\star$ will denote the 2-D convolution product

$$
(f \star g)(\vec{x})=\int_{\mathbb{R}^{2}} d^{2} \vec{y} f(\vec{x}-\vec{y}) g(\vec{y})
$$

this operation being well-defined for $f \in L^{p}$ and $g \in L^{q}$ by virtue of Young's convolution inequality [8]

$$
\|f \star g\|_{r} \leq\|f\|_{p}\|g\|_{q}, \quad 1+\frac{1}{r}=\frac{1}{p}+\frac{1}{q}
$$

where $\|\cdot\|_{p}$ stands for the norm in $L^{p}\left(\mathbb{R}^{2}, d^{2} \vec{x}\right)$. In this paper, we mean by 2-D wavelet an integrable or square integrable complex-valued function of the 2-D real variable $\vec{x}$ that has $N$ vanishing moments

$$
\iint_{\mathbb{R}^{2}} x^{p} y^{q} \psi(x, y) d x d y=0, \quad 0 \leq p, q<N .
$$

Wavelets are thus orthogonal to functions of the form

$$
P(x, y)=\sum_{k=0}^{N-1} \sum_{\ell=0}^{N-1} \gamma_{k, \ell} x^{k} y^{\ell}
$$

which means that they are blind to polynomials of order up to $N-1$ in the $x$ and $y$ variables.

Let us now recall the definition of directional wavelets given in [9].

Definition 1: A wavelet $\psi$ is said to be directional if and only if its Fourier transform is strictly supported in a convex cone with apex at the origin.

Example 1 (Cauchy Wavelets): Let $\mathcal{C}$ be the cone defined by the angles $\alpha$ and $\beta$ and let $\tilde{\alpha}=\alpha+\pi / 2, \tilde{\beta}=\beta-\pi / 2$. The Cauchy wavelet is defined by

$$
\hat{\psi}_{\ell, m}^{C}(\vec{\omega})= \begin{cases}\left(\vec{\omega} \cdot \overrightarrow{1}_{\tilde{\alpha}}\right)^{\ell}\left(\vec{\omega} \cdot \overrightarrow{1}_{\tilde{\beta}}\right)^{m} e^{-\vec{\omega} \cdot \vec{\eta}}, & \text { if } \vec{\omega} \in C \\ 0, & \text { otherwise }\end{cases}
$$

where $\ell, m \in \mathbb{N}, \overrightarrow{1}_{\alpha}$ stands for the unit vector in the direction $\alpha$ and $\vec{\eta}$ is a vector in the cone defined by the angles $\tilde{\alpha}, \tilde{\beta}$. These wavelets, introduced in [9], have fast decay in the Fourier domain and are strictly supported in $\mathcal{C}$ as can be seen in Fig. 1(a).

Example 2 (Gabor or Morlet Wavelets): Let $\vec{\omega}_{0}$ be a fixed frequency vector. The 2-D Morlet wavelet is defined by

$$
\hat{\psi}(\vec{\omega})=e^{-\left\|\vec{\omega}-\vec{\omega}_{0}\right\|^{2}} .
$$




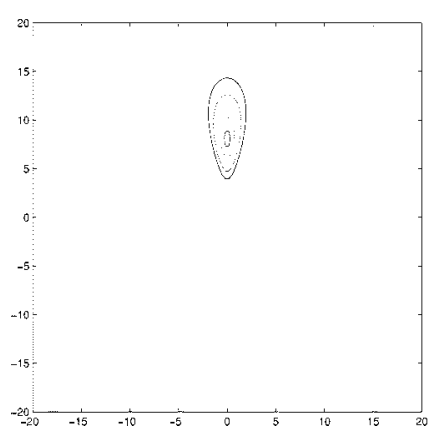

(a)

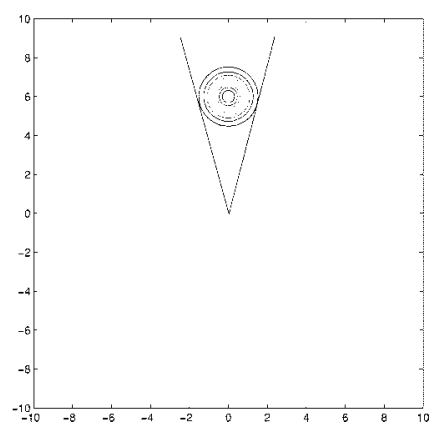

(b)
Fig. 1. Fourier transform of the (a) Cauchy and (b) Morlet wavelets.

Strictly speaking it is not a directional wavelet ${ }^{1}$ (it does not satisfy Def. 1 ), but it is numerically very close to it. This can also be verified on Fig. 1(b) where we have drawn the cone in which this wavelet is mostly concentrated.

\section{Two-Dimensional WAVELET FrameS}

In this section, we briefly review the definition and properties of translation invariant frames of wavelets also frequently called DWTs in the literature. The interested reader should refer to [3] for more details on this specific case and to [10], [11] for a deep introduction to general frame theory. We will make use of the so-called DWT and, in order to bring orientation sensitivity into this scheme, we will use $K$ oriented wavelets $\left\{\psi^{k}(\vec{x}), 1 \leq k \leq K\right\}$. By this we mean that the support of each $\hat{\psi}^{\ell}$ is essentially included in a ball around reference wave vector $\vec{k}_{\ell}$ and that the sum of these supports approximately covers an annulus in frequency space (see Fig. 2).

Denoting the scaled and translated wavelets

$$
\psi_{2^{j}, \vec{b}}^{k}(\vec{x})=\frac{1}{2^{2 j}} \psi^{k}\left(2^{-j}(\vec{x}-\vec{b})\right)
$$

the DWT of $f \in L^{2}\left(\mathbb{R}^{2}, d^{2} \vec{x}\right)$ with respect to $\psi^{k}$ is given by

$$
\mathcal{W}_{f}^{k}\left(2^{j}, \vec{b}\right)=\left\langle\psi_{2^{j}, \vec{x}}^{k}, f\right\rangle=\left(\tilde{\psi}^{k} \star f\right)(\vec{x})
$$

with $\tilde{\psi}(\vec{x})=\overline{\psi(-\vec{x})}$. The DWT is a complete and stable representation of the signal provided there exist $A>0$ and $B$ finite such that

$$
A \leq \sum_{k=1}^{K} \sum_{j \in \mathbb{Z}}\left|\hat{\psi}^{k}\left(2^{j} \vec{\omega}\right)\right|^{2} \leq B \quad \forall \vec{\omega} \in \mathbb{R}^{2} \backslash \overrightarrow{0} .
$$

In this case dual or reconstruction wavelets are defined by

$$
\hat{\chi}^{k}(\vec{\omega})=\frac{\hat{\psi}^{k}(\vec{\omega})}{\sqrt{\sum_{k} \sum_{j}\left|\hat{\psi}^{k}\left(2^{j} \vec{\omega}\right)\right|^{2}}}
$$

for which we have

$$
\sum_{j \in \mathbb{Z}} \sum_{k=1}^{K} \hat{\chi}^{k}\left(2^{j} \vec{\omega}\right) \overline{\hat{\psi}^{k}\left(2^{j} \vec{\omega}\right)}=1 .
$$

${ }^{1}$ Actually it is not even a wavelet according to (2), but the error is very small for large $\left\|\vec{\omega}_{0}\right\|$. It can be turned into a genuine wavelet by adding a suitable correcting term [6].

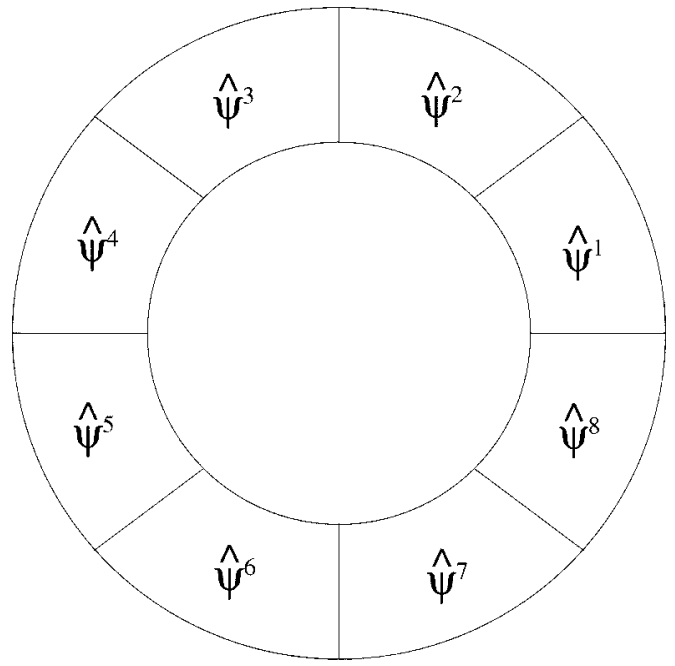

Fig. 2. Frequency localization of the oriented wavelets $\hat{\psi}^{k}$.

Finally the reconstruction formula reads

$$
f(\vec{x})=\sum_{j \in \mathbb{Z}} 2^{-2 j} \sum_{k=1}^{K}\left(\mathcal{W}_{f}^{k}\left(2^{j}, \cdot\right) \star \chi_{2^{j}}^{k}\right)(\vec{x})
$$

where the convergence of this last expression is intended in $L^{2}$ norm.

A much simpler formula can be used when condition (6) can be changed into

$$
\sum_{k=1}^{K} \sum_{j \in \mathbb{Z}} \hat{\psi}^{k}\left(2^{j} \vec{\omega}\right)=1 \quad \forall \vec{\omega} \in \mathbb{R}^{2} \backslash \overrightarrow{0} .
$$

In this case, the dual wavelets are formally Dirac distributions and the reconstruction formula then reads

$$
f(\vec{x})=\sum_{j \in \mathbb{Z}} \sum_{k=1}^{K} 2^{-j} \mathcal{W}_{f}^{k}\left(2^{j}, \vec{x}\right)
$$

that is, a simple sum of DWT coefficients with convergence in $L^{2}$ norm.

In the following, we will be a little bit more restrictive by imposing that the wavelets $\psi^{k}$ are rotated versions of a prototype directional wavelet $\psi$, that is

$$
\psi^{k}(\vec{x})=\psi\left(r_{-\theta_{k}} \vec{x}\right)
$$

where $r_{\theta}$ is a rotation matrix and $\theta_{k}=(k-1) 2 \pi / K$. The next section gives a very simple and straightforward technique for designing such frames.

\section{Custom Design of Dyadic Frames}

In this section we will briefly review the extension to 2-D of a technique introduced by Duval-Destin et al. [1] that explicitly builds dyadic wavelet frames starting from a Continuous Wavelet Transform (CWT). We proceed by first constructing frames of isotropic wavelets and we then bring angular selectivity by partitioning the mother wavelet into several directional wavelets. The main advantage of this construction is the total 
freedom obtained on the wavelet used and this allows us to really tailor the frame to our needs. We focus on the main principles and let the interested reader consult the references for clean mathematical proofs.

\section{A. Scale Discretization}

We will start from the reconstruction formula associated to the CWT in two dimensions [6]

$$
f(\vec{x})=\int_{0}^{+\infty} a^{-1} d a \int_{\mathbb{R}^{2}} d^{2} \vec{b} \mathcal{W}_{f}(a, \vec{b}) \psi_{\vec{b}, a}(\vec{x})
$$

where $\mathcal{W}_{f}(a, \vec{b})$ is the wavelet transform of the signal $f \in$ $L^{2}\left(\mathbb{R}^{2}, d^{2} \vec{x}\right)$

$$
\mathcal{W}_{f}(a, \vec{b})=C_{\psi}^{-1} a^{-2} \int_{\mathbb{R}^{2}} d^{2} \vec{x} \overline{\left(a^{-1}(\vec{x}-\vec{b})\right)} f(\vec{x})
$$

and $C_{\psi}$ is a constant

$$
C_{\psi}=2 \pi \int_{\mathbb{R}^{2}} d^{2} \vec{\omega} \frac{|\hat{\psi}(\vec{\omega})|^{2}}{\|\vec{\omega}\|^{2}}
$$

that we will normalize to one in the following. Convergence of (12) holds in $L^{2}$ norm when the wavelet $\psi \in L^{1}\left(\mathbb{R}^{2}\right)$ and $C_{\psi}<+\infty$. Note also that these equations are valid only when the wavelet $\psi$ is isotropic. ${ }^{2}$

The basic idea behind the proposed construction is now to segment the integral over scales in (12) and replace it by a sum over dyadic intervals. This is done first by rewriting the reconstruction formula

$$
f(\vec{x})=\int_{0}^{+\infty} \frac{d a}{a} d_{a}(\vec{x})
$$

where we have defined the infinitesimal detail

$$
d_{a}(\vec{x})=\int_{\mathbb{R}^{2}} d^{2} \vec{b} \mathcal{W}_{f}(a, \vec{b}) \psi_{\vec{b}, a}(\vec{x}) .
$$

By virtue of Young's convolution inequality $d_{a} \in L^{2}\left(\mathbb{R}^{2}, d^{2} \vec{x}\right)$ and, taking its Fourier transform, we obtain

$$
\hat{d}_{a}(\vec{\omega})=|\hat{\psi}(a \vec{\omega})|^{2} \hat{f}(\vec{\omega})
$$

These equations show that $d_{a}$ represents the amount of information captured by the wavelet between scales $a$ and $a+d a$, hence the name "infinitesimal details." Summing all these details, that is, integrating over the scale variable, reproduces the original signal. In the same vein, we can synthesize a low resolution approximation of $f$ by integrating up to a given resolution, say $a_{0}$

$$
f_{a_{0}}(\vec{x})=\int_{a_{0}}^{+\infty} \frac{d a}{a} d_{a}(\vec{x}) .
$$

Taking the Fourier transform on both sides suggests to introduce the following Fourier multiplier:

$$
|\hat{\phi}(\vec{\omega})|^{2}=\int_{1}^{+\infty} \frac{d a}{a}|\hat{\psi}(a \vec{\omega})|^{2} .
$$

${ }^{2}$ In the general case of anisotropic wavelets, one has to consider the action of rotations in formulas (12) and (13).
It is then shown in [1] that the approximation $f_{a}$ can be written

$$
f_{a}(\vec{x})=\int_{\mathbb{R}^{2}} d^{2} \vec{b}\left\langle\phi_{(\vec{b}, a)} \mid f\right\rangle \phi_{(\vec{b}, a)}(\vec{x})
$$

and that the following limit holds in the strong sense in $L^{2}\left(\mathbb{R}^{2}, d^{2} \vec{x}\right)$ :

$$
\lim _{a \rightarrow 0} f_{a}=f .
$$

Remark also that (16) implies

$$
\lim _{\|\vec{\omega}\| \rightarrow \infty}|\hat{\phi}(\vec{\omega})|^{2}=0
$$

and thus defines a smoothing function. Now, starting from an approximation of $f$ at scale $a=2^{J}$, we can refine up to an arbitrary resolution by adding up details. For this purpose, we introduce slices of details

$$
D_{j}(\vec{x})=\int_{2^{j}}^{2^{j+1}} \frac{d a}{a} d_{a}(\vec{x}) .
$$

Taking the Fourier transform on both sides, we have

$$
\hat{D}_{j}(\vec{\omega})=\hat{f}(\vec{\omega}) \int_{2^{j}}^{2^{j+1}} \frac{d a}{a}|\hat{\psi}(a \vec{\omega})|^{2}
$$

and this leads us to define the integrated wavelet packets

$$
|\hat{\Gamma}(\vec{\omega})|^{2}=\int_{1 / 2}^{1} \frac{d a}{a}|\hat{\psi}(a \vec{\omega})|^{2} .
$$

The name comes from the idea of gluing together wavelets but it should be stressed that it is in no way connected to the adaptive decompositions introduced by Meyer and Wickerhauser [12]. Finally, putting (17) and (19) together, we obtain the following dyadic decomposition:

$$
\begin{aligned}
f=\int_{\mathbb{R}^{2}} d^{2} \vec{b}\left\langle\phi_{\left(2^{J}, \vec{b}\right)} \mid f\right\rangle \phi_{\left(2^{J}, \vec{b}\right)} & \\
& +\sum_{j=-\infty}^{J} \int_{\mathbb{R}^{2}} d^{2} \vec{b}\left\langle\Gamma_{\left(2^{j}, \vec{b}\right)} \mid f\right\rangle \Gamma_{\left(2^{j}, \vec{b}\right)}
\end{aligned}
$$

that holds in $L^{2}$ norm. In order to simplify our notations, we will write the wavelet coefficients $\mathcal{W}_{f}\left(2^{j}, \vec{b}\right)=\left\langle\Gamma_{\left(2^{j}, \vec{b}\right)} \mid f\right\rangle$ and introduce the approximation coefficients $\mathcal{S}_{f}\left(2^{J}, \vec{b}\right)=\left\langle\phi_{\left(2^{J}, \vec{b}\right)} \mid f\right\rangle$. Equation (20) now reads

$f(\vec{x})=\left(\mathcal{S}_{f}\left(2^{J}, \cdot\right) \star \phi_{2^{J}}\right)(\vec{x})+\sum_{j=-\infty}^{J}\left(\mathcal{W}_{f}\left(2^{j}, \cdot\right) \star \Gamma_{2^{J}}\right)(\vec{x})$.

We have thus built a DWT starting from the CWT. It is important to realize that the scaling function $\phi$ and the wavelet $\Gamma$ inherit the localization and smoothness properties of $\psi$. In view of the considerable freedom we have in the choice of $\psi$, we are now able to easily design custom, translation invariant dyadic frames. A simpler decomposition formula arises when one starts from the so called Morlet reconstruction formula instead of (12):

$$
f(\vec{x})=C_{\psi}^{-1} \int_{0}^{+\infty} \frac{d a}{a} \mathcal{W}_{f}(a, \vec{x})
$$


that holds in $L^{2}$ norm for $\psi \in L^{1}\left(\mathbb{R}^{2}, d^{2} \vec{x}\right)$ and when $C_{\psi}$ is a finite constant defined by

$$
C_{\psi}=2 \pi \int_{\mathbb{R}^{2}} d^{2} \vec{\omega} \frac{\hat{\psi}(\vec{\omega})}{\|\vec{\omega}\|^{2}} .
$$

The same reasoning as before leads us to introduce a scaling function

$$
\hat{\phi}(\vec{\omega})=\int_{1}^{+\infty} \frac{d a}{a} \hat{\psi}(a \vec{\omega})
$$

and integrated wavelet packets

$$
\begin{aligned}
\hat{\Gamma}(\vec{\omega}) & =\int_{1 / 2}^{1} \frac{d a}{a} \hat{\psi}(a \vec{\omega}) \\
& =\hat{\phi}(2 \vec{\omega})-\hat{\phi}(\vec{\omega})
\end{aligned}
$$

that is, these wavelets are expressed as difference of smoothing functions. In this case, the reconstruction formula is much simpler, since it involves a straight sum of approximation and wavelet coefficients

$$
f(\vec{x})=\mathcal{S}_{f}\left(2^{J}, \vec{x}\right)+\sum_{j=-\infty}^{J} \mathcal{W}_{f}\left(2^{j}, \vec{x}\right) .
$$

One of the main advantages of this construction is that it allows to build wavelets and scaling functions that have fast decay both in the spatial and frequency domains. This is very useful in applications where one wants to use wavelets that have sharp prescribed localization properties in the Fourier domain and are also of fast decay in the spatial domain, as it is the case with Gabor functions. This is very difficult to achieve in practice. For example, if one wants to use spline-based wavelet frames, it appears that, although the spatial localization is very good, splines are not sharply localized in Fourier variables (algebraic decay) and can even show disturbing sidelobes. A concrete example is given by texture analysis where the latter are distinguished on the basis of the statistics of frequency subbands as measured using Gabor wavelets [13]. If one wants to use a dyadic frame, special care has to be given to the frequency localization of the wavelets and this can be easily done using the technique described above.

\section{B. Example}

Let us now apply the previous formalism to a concrete case. We will construct isotropic dyadic wavelets and scaling functions belonging to the class of $\mathcal{C}^{\infty}$ functions with fast decay and associated to the family of pseudo-differential operators defined by multiplication by $\omega^{n}=\left(\omega_{1}^{2}+\omega_{2}^{2}\right)^{n / 2}$ in the Fourier domain. For this purpose, we start with the family of 2-D isotropic wavelets

$$
\hat{\psi}(\vec{\omega})=\omega^{n} e^{-\omega^{2}}
$$

and we compute the associated scaling function using (16). We thus consider the family

$$
\widehat{\tilde{\phi}}_{n}(\omega)=\int_{\omega}^{+\infty} \frac{d a}{a} a^{n} e^{-a^{2}}, \quad(n \geq 2)
$$

TABLE I

SCALING FUNCTIONS OF LOWEST ORDERS

\begin{tabular}{l|l}
\hline Order & Scaling function \\
\hline$n=2$ & $\hat{\phi}_{2}(k)=e^{-k^{2}}$ \\
$n=3$ & $\hat{\phi}_{3}(k)=\frac{1}{2} k e^{-k^{2}}+\frac{\sqrt{\pi}}{4} \operatorname{erfc}(k)$ \\
$n=4$ & $\hat{\phi}_{4}(k)=e^{-k^{2}}\left(1+k^{2}\right)$ \\
$n=5$ & $\hat{\phi}_{5}(k)=\alpha_{5}^{-1}\left(\frac{1}{2} k^{3} e^{-k^{2}}+\frac{3}{4} k e^{-k^{2}}+\frac{3 \sqrt{\pi}}{8} \operatorname{erfc}(k)\right)$ \\
\hline
\end{tabular}

that satisfies the following recurrence relation:

$$
\widehat{\tilde{\phi}}_{n}(\omega)=\frac{1}{2} \omega^{n-2} e^{-\omega^{2}}+\frac{(n-2)}{2} \widehat{\tilde{\phi}}_{n-2}(\omega)
$$

with

$$
\widehat{\hat{\phi}}_{2}(\omega)=\frac{1}{2} e^{-\omega^{2}}, \quad \widehat{\tilde{\phi}}_{3}(\omega)=\frac{1}{2} \omega e^{-\omega^{2}}+\sqrt{\frac{\pi}{4}} \operatorname{erfc}(\omega)
$$

and the error function is defined by

$$
\operatorname{erfc}(\omega)=\frac{4}{\sqrt{\pi}} \int_{\omega}^{+\infty} d \omega e^{-\omega^{2}}
$$

We would like to normalize this family in such a way that $\int_{\mathbb{R}} d^{2} \vec{x} \phi_{n}(\vec{x})=1$ and this leads us to define

$$
\hat{\phi}_{n}(\omega)=\alpha_{n}^{-1} \widehat{\widetilde{\phi}}(\omega)
$$

where the constant $\alpha_{n}$ is defined by

$$
\begin{aligned}
\alpha_{n} & =\widehat{\tilde{\phi}}_{2}(0) \prod_{i=1}^{(n-2) / 2} \frac{n-2 i}{2}, n \text { even, } n>2 \\
& =\widehat{\tilde{\phi}}_{3}(0) \prod_{i=1}^{(n-3) / 2} \frac{2 i+1}{2}, n \text { odd, } \quad n>3 .
\end{aligned}
$$

The recursion is started with $\alpha_{2}=\widehat{\tilde{\phi}}_{2}(0)$ and $\alpha_{3}=\widehat{\tilde{\phi}}_{3}(0)$. Scaling functions of the lowest orders are listed in Table I. Using (19) or (26), we obtain the desired family of isotropic wavelets. Note that the parameter $n$ also controls the number of vanishing moments of the associated wavelet. An example of such a scaling function of order 4 is given on Fig. 4.

\section{Directional Wavelet Frames}

The next step in our construction is to build directional wavelet frames. A simple and straightforward way to achieve this is to start from an isotropic integrated wavelet $\Gamma$ and segment it into directional ones. This can be done by introducing an angular window $\eta(\varphi), \varphi \in[0,2 \pi]$ in the Fourier domain and then defining a new wavelet

$$
\hat{\Psi}(\omega, \varphi)=\hat{\Gamma}(\omega) \eta(\varphi) .
$$

Note that this construction amounts to work with wavelets that are separable in polar coordinates. The choice of the angular window is restricted by the reconstruction formula (9). More precisely if one makes use of (21), $\eta$ has to satisfy

$$
\sum_{k=0}^{K-1}\left|\eta\left(\varphi-\frac{2 \pi k}{K}\right)\right|^{2}=1
$$




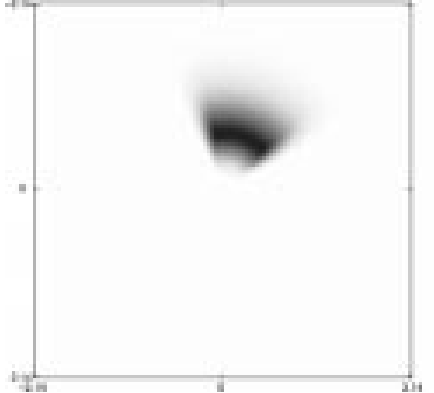

(a) (b)

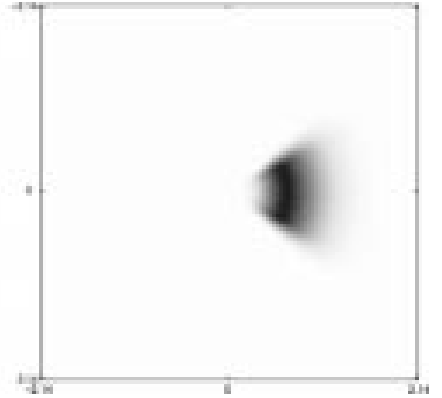

Fig. 3. Fourier transform of a directional dyadic wavelet of order 4 and angular resolution of $\pi / 5$ for the value (a) $k=0$ and (b) $k=1$ of the rotation parameter.

while the simpler formula (27) requires

$$
\sum_{k=0}^{K-1} \eta\left(\varphi-\frac{2 \pi k}{K}\right)=1
$$

where we have assumed $K$ orientations. An additional requirement, when working with directional wavelets, is that the support of $\eta$ be strictly less than $\pi$. In order to preserve the frequency localization of $\hat{\Gamma}$, it is important that $\eta$ be regular enough. The optimal choice is thus to build a partition of the circle using a suitable compactly supported $\mathcal{C}^{\infty}$ function. In Appendix A we propose a very simple construction that uses Schwartz's function. An example of dyadic directional wavelet built using this technique is depicted on Figs. 3 and 4.

\section{IMPLEMENTATION USING APPROXIMATE QMFS}

One of the main drawbacks of these oriented frames is that they are not designed to be implemented using a pyramidal algorithm. Nevertheless we will now show that one can design special QMF pairs that allow for a very good approximation of the DWT and provide a substantial gain in computation speed. Most of the material in this section is an extension to the 2-D case of the work of Muschietti and Torrésani [2], [14].

\section{A. Filter Design}

As we work with translation invariant frames, we will now sample the position parameter of the DWT over a regular grid, that is, we will consider wavelets and scaling functions indexed by the integer grid

$\Psi_{j ; m, n}^{k}(x, y)=2^{-2 j} \Psi^{k}\left(2^{-j}(x-m, y-n)\right), \quad j, m, n \in \mathbb{Z}$.

We then obtain a discrete DWT by just restricting the DWT to this particular grid

$\mathcal{W}_{j}^{k} f(m, n)=\left\langle\Psi_{j ; m, n}^{k} \mid f\right\rangle, \quad \mathcal{S}_{j} f(m, n)=\left\langle\phi_{j ; m, n} \mid f\right\rangle$.

Computing a regularly sampled continuous wavelet transform is a problem that has been studied first by Holschneider et al. [15]. Let us assume there exists 2-D discrete filters $h$ and $g^{k}$,

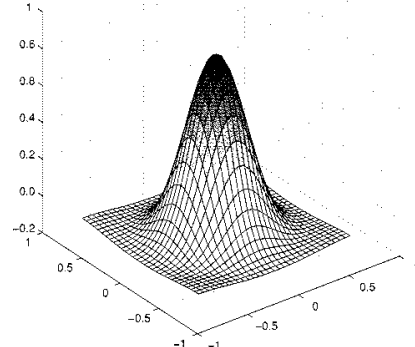

(a)

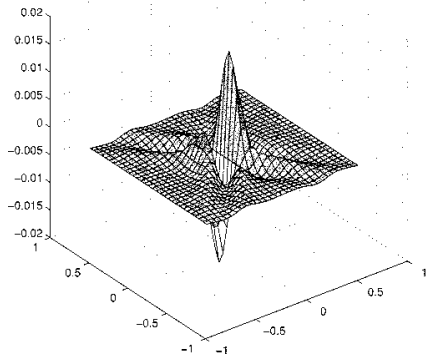

(c)

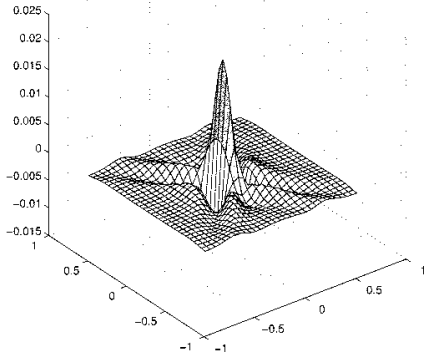

(b)

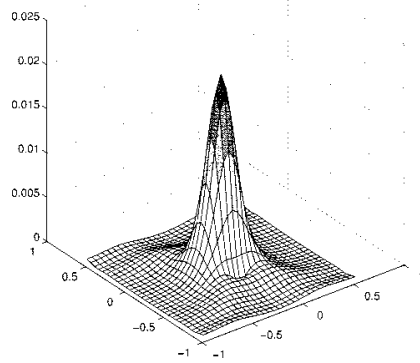

(d)
Fig. 4. Scaling function of (a) order 4, (b) real part, (c) imaginary part, and (d) modulus of the associated wavelet with angular resolution of $\pi / 5$.

$k=0 \ldots K-1$, such that one can compute these quantities using a pyramidal algorithm [16], that is

$$
\begin{aligned}
\mathcal{S}_{j} f(m, n)= & \sum_{p, q \in \mathbb{Z}} \overline{h_{p, q}} \mathcal{S}_{j-1} \\
& \cdot f\left(m-2^{j-1} p, n-2^{j-1} q\right) \\
\mathcal{W}_{j}^{k} f(m, n)= & \sum_{p, q \in \mathbb{Z}} \overline{g_{p, q}^{k}} \mathcal{S}_{j-1} \\
& \cdot f\left(m-2^{j-1} p, n-2^{j-1} q\right) .
\end{aligned}
$$

This is equivalent to asking that the related wavelets and scaling function satisfy a two-scale equation of the form

$$
\begin{aligned}
\hat{\phi}\left(2 \omega_{x}, 2 \omega_{y}\right) & =m_{0}\left(\omega_{x}, \omega_{y}\right) \hat{\phi}\left(\omega_{x}, \omega_{y}\right) \\
\hat{\Psi}^{k}\left(2 \omega_{x}, 2 \omega_{y}\right) & =m_{1}^{k}\left(\omega_{x}, \omega_{y}\right) \hat{\Psi}^{k}\left(\omega_{x}, \omega_{y}\right)
\end{aligned}
$$

where $m_{0}$ and the $m_{1}^{k}, k=0 \ldots K-1$, are the Fourier series of the filters $h$ and $g^{k}$ respectively. Relying on usual filter bank theory, we know that the same filters can be used to reconstruct the signal provided they satisfy a QMF relation

$$
\left|m_{0}\left(\omega_{x}, \omega_{y}\right)\right|^{2}+\sum_{k=0}^{K-1}\left|m_{1}^{k}\left(\omega_{x}, \omega_{y}\right)\right|^{2}=2 .
$$

This is the exact discrete equivalent of (21). Similarly, one can design filters that implement the weaker formula (27) provided they satisfy a simpler constraint

$$
m_{0}\left(\omega_{x}, \omega_{y}\right)+\sum_{k=0}^{K-1} m_{1}^{k}\left(\omega_{x}, \omega_{y}\right)=2 .
$$

The main problem at this point is that the integrated directional wavelets we have designed in Section III do not generally satisfy any two-scale equation. Another way to coin the problem 
is to remark that, although there usually exists regular multipliers $\mu_{0}$ and $\mu_{1}^{k}$ satisfying

$$
\hat{\phi}(2 \vec{\omega})=\mu_{0}(\vec{\omega}) \hat{\phi}(\vec{\omega}), \quad \hat{\Psi}^{k}(2 \vec{\omega})=\mu_{1}^{k}(\vec{\omega}) \hat{\phi}(\vec{\omega})
$$

these are not $2 \pi \times 2 \pi$ periodic and cannot be used to compute successive approximations and details as in (32) and (33). Nevertheless it is crucial to notice that these multipliers, as well as filters $m_{0}$ and $m_{1}^{k}$, are always multiplied by $\hat{\phi}$. Now, if the latter is very well localized in $[-\pi, \pi] \times[-\pi, \pi]$ and this is exactly the case we are considering in this paper, the lack of periodicity of $\mu_{0}$ and $\mu_{1}^{k}$ is compensated by the localization of $\hat{\phi}$ and it seems then reasonable to look for good approximations of $\mu_{i}$ using discrete filters. For this purpose, let us introduce the subset of $L^{2}\left(\mathbb{R}^{2}, d^{2} \vec{x}\right)$ spanned by the integer translates of the scaling function defined in (16)

$\mathcal{V}_{0}=\left\{f \in L^{2} \mid f=\sum_{m, n \in \mathbb{Z}} c_{m, n} \phi(x-m, y-n), c_{m, n} \in \ell^{2}\right\}$

and suppose that the family $\{\phi(x-m, y-n), m, n \in \mathbb{Z}\}$ forms a Riesz basis of $\mathcal{V}_{0}$. Finding a best approximant for $\mu_{0}$ can be formulated as finding an element of $\mathcal{V}_{0}$ whose distance to $(1 / 4) \phi(x / 2, y / 2)$ is minimal in $L^{2}\left(\mathbb{R}^{2}, d^{2} \vec{x}\right)$. Similarly, finding best approximants for the $\mu_{1}^{k}$ is equivalent to finding those elements of $\mathcal{V}_{0}$ that minimize the distance to $(1 / 4) \Psi^{k}(x / 2, y / 2)$. Now since $\mathcal{V}_{0}$ is a linear subspace of a Hilbert space, the projection theorem applies and guarantees the existence and uniqueness of such solutions. Suppose that $\gamma \in \mathcal{V}_{0}$ is the solution for $\mu_{0}$. It can be expanded as

$$
\gamma=\sum_{m, n \in \mathbb{Z}} h_{m, n}^{a} \phi_{m, n}
$$

where the $h_{m, n}^{a}$ are the Fourier coefficients of the approximate filter

$$
m_{0}^{a}\left(\omega_{x}, \omega_{y}\right)=\frac{1}{4 \pi^{2}} \sum_{m, n \in \mathbb{Z}} h_{m, n}^{a} e^{-i\left(m \omega_{x}+n \omega_{y}\right)}
$$

The projection theorem gives also the following characterization of these coefficients

$$
\begin{aligned}
& \iint_{\mathbb{R}^{2}} d x d y( \frac{\phi(x+m, y+n)}{} \\
&\left.\quad \times\left[\sum_{p, q \in \mathbb{Z}} h_{p, q}^{a} \phi(x+p, y+q)-\frac{1}{4} \phi\left(\frac{x}{2}, \frac{y}{2}\right)\right]\right) \\
&=0, \forall m, n \in \mathbb{Z} .
\end{aligned}
$$

Similarly, for the directional wavelets, one obtains $K$ approximate filters $g_{m, n}^{k, a}$ characterized by

$$
\begin{aligned}
\iint_{\mathbb{R}^{2}} d x d y( & \frac{\phi(x+m, y+n)}{\phi} \\
\times & {\left.\left[\sum_{p, q \in \mathbb{Z}} g_{p, q}^{k, a} \phi(x+p, y+q)-\frac{1}{4} \Psi^{k}\left(\frac{x}{2}, \frac{y}{2}\right)\right]\right) } \\
=0, & \forall m, n \in \mathbb{Z} .
\end{aligned}
$$

Finally, the approximate low resolution and detail coefficients read

$$
\begin{aligned}
\mathcal{S}_{j}^{a} f(m, n)= & \sum_{p, q \in \mathbb{Z}} \overline{h_{p, q}^{a}} \mathcal{S}_{j-1}^{a} \\
& \cdot f\left(m-2^{j-1} p, n-2^{j-1} q\right) \\
\text { and } & \\
\mathcal{W}_{j}^{k, a} f(m, n)= & \sum_{p, q \in \mathbb{Z}} \overline{g_{p, q}^{k, a}} \mathcal{S}_{j-1}^{a} \\
& \cdot f\left(m-2^{j-1} p, n-2^{j-1} q\right) .
\end{aligned}
$$

The following theorem, which is a 2-D extension of the result found in [2], gives us explicit formulas for the best approximants $m_{0}^{a}$ and $m_{1}^{k, a}$, as well as an estimation of the error with respect to the original coefficients $\mathcal{S}_{j} f(m, n)$ and $\mathcal{W}_{j}^{k} f(m, n)$.

\section{Theorem 1 (Muschietti and Torrésani):}

i) The filters $m_{0}^{a}$ et $m_{1}^{k, a}$ solutions of (36) and (37) are given by the equation shown at the bottom of the page.

ii) If we write ${ }^{3} C_{i}=2 \operatorname{ess} \sup _{\vec{\omega} \in \mathbb{R}^{2}} m_{i}^{a}(\vec{\omega}), i=0,1$ and

$$
\nu\left(m_{i}, m_{i}^{a}\right)=\left[\iint_{\mathbb{R}^{2}} d^{2} \vec{\omega}\left|m_{i}^{a}(\vec{\omega})-\mu_{i}(\vec{\omega})\right||\hat{\phi}(\vec{\omega})|^{2}\right]^{1 / 2}
$$

then

$$
\begin{aligned}
&\left\|\mathcal{S}_{j}^{a} f-\mathcal{S}_{j} f\right\|_{\infty} \leq 2 \nu\left(m_{0}^{a}, \mu_{0}\right) \cdot \frac{1-\left(C_{0}\right)^{j}}{1-C_{0}}\|f\|_{2}, \\
&\left\|\mathcal{W}_{j}^{k, a} f-\mathcal{W}_{j}^{k} f\right\|_{\infty} \leq 2\left(\nu\left(m_{1}^{a}, \mu_{1}\right)+C_{1} \nu\left(m_{0}^{a}, \mu_{0}\right)\right. \\
&\left.\times \frac{1-\left(C_{0}\right)^{j-1}}{1-C_{0}}\right)\|f\|_{2} .
\end{aligned}
$$

For completeness, a proof of this result is given in the Appendix.

\footnotetext{
${ }^{3}$ Since this quantity is orientation independent, we drop the corresponding superscript for ease of notation.
}

$$
\begin{aligned}
m_{0}^{a}\left(\omega_{x}, \omega_{y}\right) & =\frac{\sum_{p, q \in \mathbb{Z}} \hat{\phi}\left(2\left(\omega_{x}+2 \pi p\right), 2\left(\omega_{y}+2 \pi q\right)\right) \overline{\hat{\phi}\left(\omega_{x}+2 \pi p, \omega_{y}+2 \pi q\right)}}{\sum_{p, q \in \mathbb{Z}}\left|\hat{\phi}\left(\omega_{x}+2 \pi p, \omega_{y}+2 \pi q\right)\right|^{2}} \\
m_{1}^{k, a}\left(\omega_{x}, \omega_{y}\right) & =\frac{\sum_{p, q \in \mathbb{Z}} \hat{\Psi}^{k}\left(2\left(\omega_{x}+2 \pi p\right), 2\left(\omega_{y}+2 \pi q\right)\right) \overline{\hat{\phi}\left(\omega_{x}+2 \pi p, \omega_{y}+2 \pi q\right)}}{\sum_{p, q \in \mathbb{Z}}\left|\hat{\phi}\left(\omega_{x}+2 \pi p, \omega_{y}+2 \pi q\right)\right|^{2}}
\end{aligned}
$$




\section{B. Implementation Issues}

When implementing (38) and (39), the first fact to consider is that, generally, we possess only a finite number $M \times N$ of samples $f(m, n)$ with $(m, n) \in[0 \ldots M-1,0 \ldots N-1]$. A decision has thus to be made about the nature of the signal outside this range.

Furthermore, the filters will practically be computed on a finite grid. That is, we will only use a finite number $P \times Q$ of filter coefficients $h_{p, q}^{a}$ and $g_{p, q}^{k, a}$ (with $(p, q) \in[-P / 2 \ldots P / 2-$ $1,-Q / 2 \ldots Q / 2-1])$.

In a first approach, the signal is considered to be zero outside this range. Unfortunately, this decision leads to impractical and inefficient algorithms, as one has to compute $\left(M+2^{j-1}(P-\right.$ 1)) $\times\left(N+2^{j-1}(Q-1)\right)$ for each $j>0$ to avoid side-effects.

A second approach is to consider the signal as being periodic of period $M \times N$. With this approach, fast circular convolution algorithms can be used and we are led to the following algorithm structure:

1) compute $m_{0}^{a}$ and $m_{1}^{k, a}$ using Theorem 1 and the associated impulse responses;

2) compute a first approximation of the analyzed signal $f$ using (31); this step is traditionally skipped and the signal is considered as its first approximation;

3 ) for each $j>0$, iteratively compute details and approximations using (38) and (39).

The cost of this algorithm is

$$
C(M, N)=c \cdot M \cdot N
$$

where the constant $c$ depends on the size of the impulse responses of the filters. This dependency strongly limits the power of this algorithm as experiments show that, even for small sizes, this algorithm is always slower than the FFT based algorithm used to compute the CWT. As those sizes may not be arbitrarily choosen, another algorithm should be used in order to get valuable results with small computation times.

The obvious way to handle this problem is to replace convolutions in the real space by products of Fourier transforms in the frequency space. The main advantage of this technique is that we no longer need to restrict ourselves to use small filters, as we may now use impulse responses that are the same size as the signal, thus giving much more precise results than with the above algorithm. For the sake of simplicity, we will develop this new algorithm in the 1-D case, its extension to the 2-D directional case being straightforward.

For the first step, we have to compute two periodic convolution products

$$
\begin{aligned}
c_{-1, k} & =\sum_{n=0}^{N-1} c_{0, k-n} h_{n} \\
d_{-1, k} & =\sum_{n=0}^{N-1} c_{0, k-n} g_{n} .
\end{aligned}
$$

By introducing $\left\{C_{j, n}\right\}=F F T_{N}\left(\left\{c_{j, k}\right\}\right),\left\{D_{j, n}\right\}=$ $F F T_{N}\left(\left\{d_{j, k}\right\}\right),\left\{H_{n}\right\}=F F T_{N}\left(\left\{h_{k}\right\}\right)$ and $\left\{G_{n}\right\}=$ $F F T_{N}\left(\left\{g_{k}\right\}\right)$ where the notation $F F T_{N}$ means the FFT algorithm applied to a sequence of length $N$, we get $C_{-1, n}=\sqrt{N} C_{0, n} H_{n}$ and $D_{-1, n}=\sqrt{N} C_{0, n} G_{n}$.
We may then get the details back in the real space with $\left\{d_{j, k}\right\}=I F F T_{N}\left(\left\{D_{j, n}\right\}\right)$.

For the second step, we have to compute

$$
\begin{aligned}
& c_{-2, k}=\sum_{n=0}^{N-1} c_{-1, k-2 n} h_{n} \\
& d_{-2, k}=\sum_{n=0}^{N-1} c_{-1, k-2 n} g_{n} .
\end{aligned}
$$

Given the periodicity of the signal, we may rewrite this as

$$
\begin{aligned}
c_{-2, k}^{0} \stackrel{\text { def }}{=} c_{-2,2 k} & =\sum_{n=0}^{N / 2-1} c_{-1,2(k-n)}\left(h_{n}+h_{n+N / 2}\right) \\
c_{-2, k}^{1} \stackrel{\text { def }}{=} c_{-2,2 k+1} & =\sum_{n=0}^{N / 2-1} c_{-1,2(k-n)+1}\left(h_{n}+h_{n+N / 2}\right) \\
d_{-2, k}^{0} \stackrel{\text { def }}{=} d_{-2,2 k} & =\sum_{n=0}^{N / 2-1} c_{-1,2(k-n)}\left(g_{n}+g_{n+N / 2}\right) \\
d_{-2, k}^{1} \stackrel{\text { def }}{=} d_{-2,2 k+1} & =\sum_{n=0}^{N / 2-1} c_{-1,2(k-n)+1}\left(g_{n}+g_{n+N / 2}\right)
\end{aligned}
$$

for $k=0, \ldots, N / 2-1$. Let us now define $h_{n}^{1}=h_{n}+h_{n+N / 2}$ for $n=0, \ldots, N / 2-1$, we get

$$
\begin{aligned}
& c_{-2, k}^{0}=\sum_{n=0}^{N / 2-1} c_{-1, k-n}^{0} h_{n}^{1} \\
& c_{-2, k}^{1}=\sum_{n=0}^{N / 2-1} c_{-1, k-n}^{1} h_{n}^{1} \\
& d_{-2, k}^{0}=\sum_{n=0}^{N / 2-1} c_{-1, k-n}^{0} g_{n}^{1} \\
& d_{-2, k}^{1}=\sum_{n=0}^{N / 2-1} c_{-1, k-n}^{1} g_{n}^{1}
\end{aligned}
$$

where $c_{-1, k}^{0}=c_{-1,2 k}, c_{-1, k}^{1}=c_{-1,2 k+1}, d_{-1, k}^{0}=d_{-1,2 k}$ and $d_{-1, k}^{1}=d_{-1,2 k+1}$. In the Fourier space, (40) and (41) may be rewritten as

$$
\begin{aligned}
C_{-2, n}^{0} & =\sqrt{\frac{N}{2}} C_{-1, n}^{0} H_{n}^{1} & C_{-2, n}^{1} & =\sqrt{\frac{N}{2}} C_{-1, n}^{1} H_{n}^{1} \\
D_{-2, n}^{0} & =\sqrt{\frac{N}{2}} C_{-1, n}^{0} G_{n}^{1} & D_{-2, n}^{1} & =\sqrt{\frac{N}{2}} C_{-1, n}^{1} G_{n}^{1}
\end{aligned}
$$

where $\left\{C_{-2, n}^{0}\right\}=F F T_{N / 2}\left(\left\{c_{-2, k}^{0}\right\}\right),\left\{C_{-2, n}^{1}\right\}=$ $F F T_{N / 2}\left(\left\{c_{-2, k}^{1}\right\}\right), \quad\left\{D_{-2, n}^{0}\right\}=F F T_{N / 2}\left(\left\{d_{-2, k}^{0}\right\}\right)$ and $\left\{D_{-2, n}^{1}\right\}=F F T_{N / 2}\left(\left\{d_{-2, k}^{1}\right\}\right)$. A straightforward calculation gives

$$
H_{n}^{1}=\sqrt{2} H_{2 n}
$$

for $n=0, \ldots, N / 2-1$. Furthermore

$$
\begin{aligned}
& C_{-1, n}^{0}=\frac{1}{\sqrt{2}}\left(C_{-1, n}+C_{-1, n+N / 2}\right) \\
& C_{-1, n}^{1}=\frac{e^{2 i \pi n / N}}{\sqrt{2}}\left(C_{-1, n}-C_{-1, n+N / 2}\right)
\end{aligned}
$$




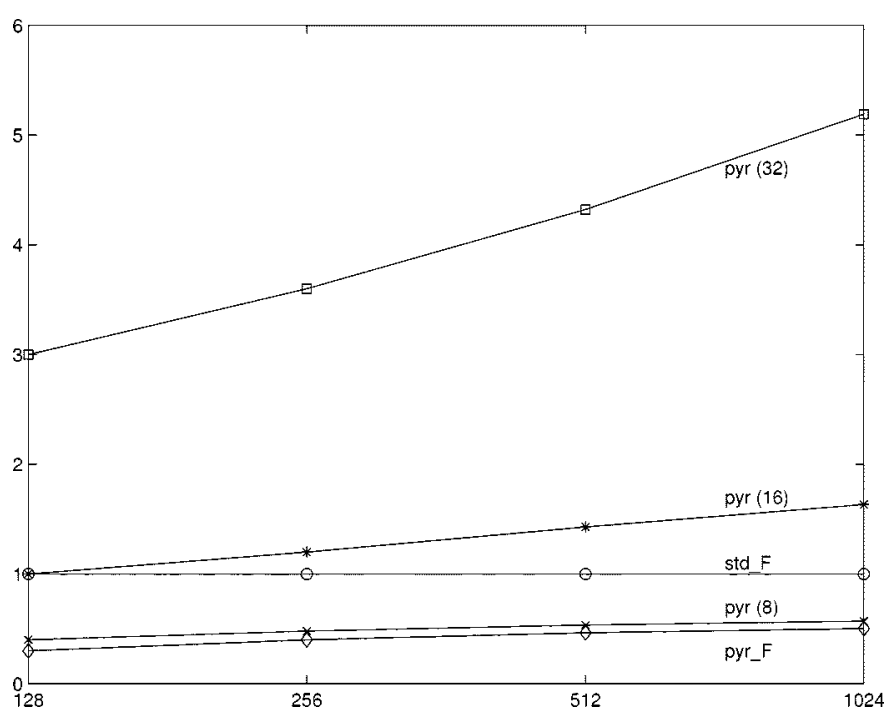

Fig. 5. Computation time for different implementations of the 2-D pyramidal algorithm as a function of the image width (square images assumed): $\operatorname{pyr}(n)$ is the standard pyramidal algorithm with $n$ by $n$ filters, pyr $F$ is the modified Fourier pyramidal algorithm explained in the text. The graph is normalized with respect to the standard algorithm with convolution in the Fourier domain $\left(\operatorname{std} \_F\right)$.

for $n=0, \ldots, N / 2-1$. The last equation is particularly interesting as it makes appear the same twiddle factors already present in the traditional FFT implementations.

Extending the above results to the following steps, one comes to the conclusion that the complexity of this new algorithm is also of order $N \log _{2}(N)$, but with a hidden constant that is exactly half the one encountered in the FFT based algorithm, this constant being associated with the pyramidal structure. The same ideas apply to the 2-D case, thus giving an algorithm whose complexity is

$$
C(M, N)=M \cdot N \cdot \log _{2}(M . N)
$$

with a hidden constant also exactly half that of the FFT based algorithm. As a matter of comparison, Fig. 5 shows timings of this algorithm for different image sizes. Timings of the usual pyramidal algorithm and standard implementation in the Fourier domain are also displayed.

Putting it all together, we have a new fast algorithm, perfectly suited to compute the 2-D CWT, faster than the traditional "pseudo-pyramidal" algorithm and sharper. Furthermore, it is essential to note that the whole construction is equivalent to that leading to the FFT algorithm in the Fourier transform theory. It should be noted that a Fourier implementation of the pyramidal algorithm is quite natural when one adresses the problem of designing maximally regular wavelets. That is why the algorithm described above shares common features with the Fourier implementation of the Meyer wavelet decomposition [17]. We refer the interested reader to the work of Rioul and Duhamel [18] for more general considerations on implementating pyramidal algorithms in the frequency domain.

\section{Alternative Implementations}

In this section, we will briefly review other techniques that would allow one to achieve directional sensitivity within a DWT framework.
Probably the most successful design of orientation sensitive decomposition is Freeman and Adelson's construction of steerable filters [19]. These filters implement a special type of oriented wavelet-like decomposition that shares with our construction the idea of polar separability in the Fourier domain. While the radial frequency part of the filters is simply constrained by the usual subband splitting and reconstruction relations, the angular part undergoes a more complicated design procedure. In essence, the goal is to be able to synthesize any orientation of a prototype waveform on basis of a few rotated versions of itself. This is very useful in specific applications where one would like to analyze images at all orientations without increasing the total computational cost of the algorithms. Such examples can be found in [19]-[21]. The main problems or difficulties that arise with steerable filters are due to these angular constraints that make design much more tricky than in the present approach. Since the implementation itself is exactly the same as the one described here, we would say that steerable filters are the preferred solution when one really makes use of the steerable property, that is, when analyzing at all orientations is needed.

Another technique that allows to easily design application specific wavelets was introduced by Abry and Aldroubi [22]. The results obtained by this approach are very powerful, but the authors have mainly focused on (bi)orthogonal wavelets and have not considered 2-D nonseparable frames.

Finally, several authors have advocated the use of splines to approximate wavelet frames decompositions [23]. This approach leads to low complexity algorithms and very efficient results when one is interested in analyzing singularities (e.g., edges) in images. Nevertheless, when dealing with the analysis of frequencies in images, the approximation may give bad results due the poor frequency localization of splines.

\section{CONCLUSIONS}

We have introduced a method to design and implement 2-D translation invariant frames of directional wavelets. This scheme is caracterized by weak design constraints that allow one to tailor wavelets to specific needs: fast decay in the spatial and frequency domains, prescribed frequency localization or orientation selectivity, etc. The interest of such decompositions resides in the processing of images considered as real 2-D objects which can be more efficiently represented by real 2-D atoms and not just tensor products of 1-D basis. With this in mind it is reasonable to argue that translations, scalings and rotations are preferred geometric operations that apply to images, hence the usefulness of efficient atomic representations involving these similitudes of $\mathbb{R}^{2}$. This technique has already found successful applications in texture analysis and synthesis, watermarking [24] or image quality assessment [25] for example.

\section{APPENDIX A}

\section{EXAMPLE OF ANGULAR DECOMPOSITION}

Let us consider the following $\mathcal{C}^{\infty}$, compactly supported, function of one real variable (Schwartz's function)

$$
g_{a}(x)= \begin{cases}e^{-\sigma^{2} /\left(a^{2}-x^{2}\right)}, & \text { if }-a \leq x \leq a \\ 0, & \text { otherwise. }\end{cases}
$$


For a given $\tau \in \mathbb{R}_{*}^{+}$, let $d_{\tau}(x)$ stand for the periodization of $g$

$$
d_{\tau}(x)=\sum_{n \in \mathbb{Z}} g_{a}(x-n \tau)
$$

By carefully choosing $a$ and $\sigma$ in (42), we can guarantee that $d_{\tau}$ is strictly positive definite. Introducing

$$
w_{\tau}(x)=\frac{g_{a}(x)}{d_{\tau}(x)}
$$

it is easy to check that it is a $\mathcal{C}^{\infty}$, compactly supported, function. Furthermore, it satisfies

$$
\sum_{n \in \mathbb{Z}} w_{\tau}(x-n \tau)=1, \quad \forall x \in \mathbb{R} .
$$

Now taking $a<\pi / 2, \tau=\pi / K$ and periodizing $w_{\tau}$, we end up with a $\mathcal{C}^{\infty}$ partition of the unit circle

$$
\begin{array}{r}
\eta(x)=\sum_{m \in \mathbb{Z}} w_{\pi / K}(x-2 m \pi) \\
\text { and } \\
\sum_{k=0}^{K-1} \eta\left(x-\frac{k \pi}{K}\right)=1 .
\end{array}
$$

This is illustrated on Fig. 6 where we have plotted these functions.

\section{APPENDIX B}

\section{PROOF OF THEOREM 1}

We will essentially follow the proof given in [2]. The first part is obtained by taking the Fourier transform of (36) and (37) and using the periodicity of $m_{0}^{a}$ and $m_{1}^{k, a}$. We then have

$$
\begin{aligned}
\int_{0}^{2 \pi} d \omega_{x} \int_{0}^{2 \pi} d \omega_{y} e^{i \omega_{x} m+i \omega_{y} n} \\
2\left\{m_{0}^{a}\left(\omega_{x}, \omega_{y}\right) \sum_{k, l \in \mathbb{Z}}\left|\hat{\phi}\left(\omega_{x}+2 \pi k, \omega_{y}+2 \pi l\right)\right|^{2}\right. \\
\quad-\sum_{k, l \in \mathbb{Z}} \frac{\hat{\phi}\left(\omega_{x}+2 \pi k, \omega_{y}+2 \pi l\right) \hat{\phi}}{} \\
\left.\cdot\left(2\left(\omega_{x}+2 \pi k\right), 2\left(\omega_{y}+2 \pi l\right)\right)\right\}=0
\end{aligned}
$$

which gives the result for $m_{0}^{a}$ and similarly for $m_{1}^{k, a}$. As for the second part of the theorem, the inequality $\|f\|_{\infty} \leq\|\hat{f}\|_{1} / 4 \pi^{2}$ allows us to work directly in the Fourier domain. Let us introduce the following quantities:

$$
\begin{aligned}
\tilde{\mathcal{S}}_{j}(m, n) & =\sum_{k, l \in \mathbb{Z}} \overline{h_{k, l}^{a}} \mathcal{S}_{j-1}\left(m-2^{j-1} k, n-2^{j-1} l\right), \\
\tilde{\mathcal{W}}_{j}^{k}(m, n) & =\sum_{p, q \in \mathbb{Z}} \overline{g_{p, q}^{k, a}} \mathcal{S}_{j-1}\left(m-2^{j-1} p, n-2^{j-1} q\right) .
\end{aligned}
$$

We have the inequality

$$
\left\|\widehat{\mathcal{S}_{j}^{a}}-\widehat{\mathcal{S}_{j}}\right\|_{1} \leq\left\|\widehat{\mathcal{S}_{j}^{a}}-\widehat{\tilde{\mathcal{S}}_{j}}\right\|_{1}+\left\|\widehat{\widetilde{\mathcal{S}}_{j}}-\widehat{\mathcal{S}_{j}}\right\|_{1}
$$

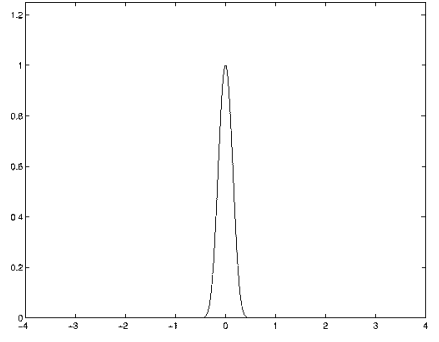

(a)

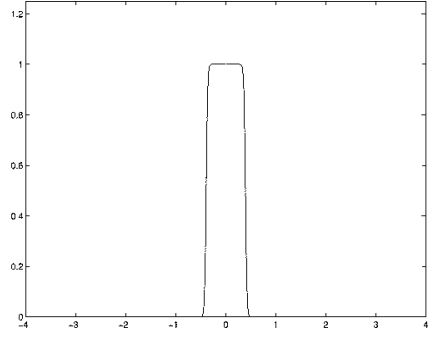

(b)

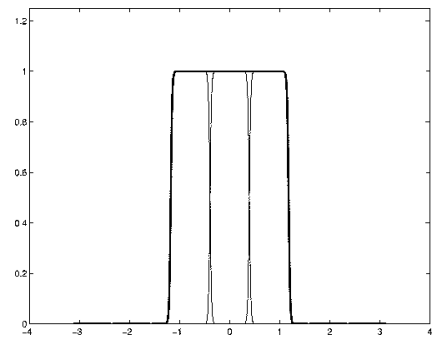

(c)

Fig. 6. Schwartz's function (a) $g_{\pi / 8}$ and (b) the corresponding angular window $\eta$. (c) Three such translated windows and their sum (thick line).

and similarly for $\left\|\widehat{\mathcal{W}_{j}^{k, a}}-\widehat{\mathcal{W}_{j}^{k}}\right\|_{1}$. Let us now compute the second term in the r.h.s of (48)

$$
\begin{aligned}
\left\|\widehat{\hat{\mathcal{S}}_{j}}-\widehat{\mathcal{S}_{j}}\right\|_{1}=\int_{\mathcal{S}^{1} \times S^{1}} d \omega_{x} d \omega_{y} 2^{j} \\
\mid \sum_{k, l \in \mathbb{Z}} \hat{f}\left(\omega_{x}+2 \pi k, \omega_{y}+2 \pi l\right) \hat{\phi} \\
\quad \times\left(2^{j}\left(\omega_{x}+2 \pi k\right), 2^{j}\left(\omega_{y}+2 \pi l\right)\right) \\
\quad-\sum_{k, l \in \mathbb{Z}} \hat{f}\left(\omega_{x}+2 \pi k, \omega_{y}+2 \pi l\right) \\
\quad \times m_{0}^{a}\left(2^{j-1} \omega_{x}, 2^{j-1} \omega_{y}\right) \\
\quad \times \hat{\phi}\left(2^{j-1}\left(\omega_{x}+2 \pi k\right), 2^{j-1}\left(\omega_{y}+2 \pi l\right)\right) \mid .
\end{aligned}
$$

Using the Cauchy-Schwarz inequality and by periodicity of $m_{0}^{a}$, we find

$$
\begin{aligned}
\left\|\widehat{\hat{\mathcal{S}}_{j}}-\widehat{\mathcal{S}_{j}}\right\|_{1} & \leq 8 \pi^{2}\|f\|_{2} \sqrt{\int_{\mathbb{R}^{2}} d^{2} \vec{\omega}\left|m_{0}^{a}(\vec{\omega}) \hat{\phi}(\vec{\omega})-\hat{\phi}(2 \vec{\omega})\right|^{2}} \\
& \leq 8 \pi^{2}\|f\|_{2} \nu\left(m_{0}^{a}, \mu_{0}\right)
\end{aligned}
$$

For the first term at the r.h.s of (48), we find

$$
\begin{aligned}
\left\|\widehat{\mathcal{S}_{j}^{a}}-\widehat{\tilde{\mathcal{S}}_{j}}\right\|_{1} & \leq 2 \int_{\mathbb{R}^{2}} d^{2} \vec{\omega}\left|m_{0}^{a}\left(2^{j-1} \vec{\omega}\right)\right| \widehat{\mathcal{S}_{j-1}^{a}}(\vec{\omega})-\widehat{\mathcal{S}_{j-1}}(\vec{\omega}) \mid \\
& \leq 2 \sup _{\vec{\omega} \in \mathbb{R}^{2}} \operatorname{ess}\left|m_{0}^{a}\right| \mid \widehat{\mathcal{S}_{j-1}^{a}}-\widehat{\mathcal{S}_{j-1}} \|_{1} .
\end{aligned}
$$

Combining these estimations, we have

$$
\left\|\widehat{\mathcal{S}_{j}^{a}}-\widehat{\mathcal{S}_{j}}\right\|_{1} \leq 8 \pi^{2}\|f\|_{2} \nu\left(m_{0}^{a}, \mu_{0}\right)+C_{0}\left\|\widehat{\mathcal{S}_{j-1}^{a}}-\widehat{\mathcal{S}_{j-1}}\right\|_{(49)} .
$$

By iteratively bounding the last term of (49), in the same way, we finally obtain

$$
\left\|\widehat{\mathcal{S}_{j}^{a}}-\widehat{\hat{\mathcal{S}}_{j}}\right\|_{1} \leq 8 \pi^{2}\|f\|_{2} \nu\left(m_{0}^{a}, \mu_{0}\right) \frac{1-\left(C_{0}\right)^{j}}{1-C_{0}} .
$$


An equivalent processing of the wavelet coefficients achieves the proof.

\section{REFERENCES}

[1] M. Duval-Destin, M. A. Muschietti, and B. Torrésani, "Continuous wavelet decompositions: Multiresolution and contrast analysis," SIAM J. Math. Anal., vol. 24, pp. 739-755, 1993.

[2] M. A. Muschietti and B. Torrésani, "Pyramidal algorithms for Littlewood-Paley decompositions," SIAM J. Math. Anal., vol. 26, pp. 925-943, 1995.

[3] S. G. Mallat and S. Zhong, "Characterization of signals from multiscale edges," IEEE Trans. Pattern Anal. Machine Intell., vol. 14, pp. 710-732, July 1992.

[4] J. Lu, D. Healy, and J. B. Weaver, "Signal recovery and wavelet reproducing kernels," in Proc. IEEE Int. Symp. Time-Frequency and TimeScale Analysis, Victoria, BC, Canada, Oct. 1992, pp. 177-179.

[5] M. Unser, "Texture classification and segmentation using wavelet frames," IEEE Trans. Image Processing, vol. 11, pp. 1549-1560, Nov. 1995.

[6] J.-P. Antoine, P. Carrette, R. Murenzi, and B. Piette, "Image analysis with two-dimensional continuous wavelet transform," Signal Process., vol. 31, pp. 241-272, 1993

[7] J. G. Daugman, "Complete discrete 2-D Gabor transforms by neural networks for image analysis and compression," IEEE Trans. Acoust. Speech Signal Process., vol. 36, pp. 1169-1179, July 1988.

[8] E. M. Stein and G. Weiss, Fourier Analysis on Euclidean Spaces. Princeton, NJ: Princeton Univ. Press, 1971.

[9] J.-P. Antoine, R. Murenzi, and P. Vandergheynst, "Directional wavelets revisited: Cauchy wavelets and symmetry detection in patterns," Appl. Comput. Harmon. Anal., vol. 6, pp. 314-345, 1999.

[10] I. Daubechies, Ten Lectures on Wavelets. Philadelphia, PA: SIAM, 1992.

[11] S. Mallat, A Wavelet Tour of Signal Processing. New York: Academic, 1998.

[12] R. R. Coifman, Y. Meyer, and M. V. Wickerhauser et al., "Wavelet analysis and signal processing," in Wavelets and Their Applications, B. Ruskai et al., Eds: Jones and Bartlett, 1992, pp. 153-178.

[13] A. K. Jain and F. Farrokhnia, "Unsupervised texture segmentation using Gabor filters," Pattern Recognit., vol. 12, pp. 1167-1186, 1991.

[14] B. Torrésani, "Some remarks on wavelet decompositions and geometric aspects," in Wavelets, Theory and Applications, M. Chui and Puccio, Eds. New York: Academic, 1994, pp. 91-116.

[15] M. Holschneider, R. Kronland, J. Morlet, and P. Tchamitchian, "A real-time algorithm for signal analysis with the help of the wavelet transform," in Wavelets, Ph. Tchamitchian, J. M. Combes, and A. Grossman, Eds. Berlin, Germany: Springer-Verlag, 1989.

[16] S. G. Mallat, "A theory for multiresolution signal decomposition: The wavelet representation," IEEE Trans. Pattern Anal. Machine Intell., vol. 11, pp. 674-693, July 1989.
[17] E. Kolaczyk, "Wavelet methods for the inversion of certain homogeneous linear operators," Ph.D. dissertation, Stanford Univ., Stanford, CA, 1997.

[18] O. Rioul and P. Duhamel, "Fast algorithms for discrete and continuous wavelet transforms," IEEE Trans. Inform. Theory, vol. 2, pp. 569-586, Mar. 1992.

[19] W. T. Freeman and E. H. Adelson, "The design and use of steerable filters," IEEE Trans. Pattern Anal. Machine Intell., vol. 13, pp. 891-906, Sept. 1991.

[20] E. Simoncelli and W. T. Freeman, "The steerable pyramid: A flexible architecture for multiscale derivative computation," in Proc. 2nd IEEE Int. Conf. Image Processing, 1995.

[21] E. Simoncelli, W. T. Freeman, E. H. Adelson, and D. J. Heerger, "Shiftable multiscale transforms," IEEE Trans. Inform. Theory, vol. 38, pp. 587-607, Mar. 1992.

[22] P. Abry and A. Aldroubi, "Designing multiresolution analysis-type wavelets and their fast algorithms," J. Fourier Anal. Applicat., vol. 2, pp. 136-159, 1995.

[23] M. J. Vrhel, C. Lee, and M. Unser, "Rapid computation of the continuous wavelet transform by oblique projections," IEEE Trans. Signal Processing, vol. 45, pp. 891-900, Apr. 1997.

[24] S. Winkler and M. Kutter, "Vers un tatouage à étalement de spectre optimal utilisant le système visuel humain," in Proc. CORESA Workshop, Sophia Antipolis, France, 1999, pp. 25-33.

[25] S. Winkler and P. Vandergheynst, "Computing isotropic local contrast from oriented pyramid decompositions," in Proc. 6th IEEE Int. Conf. Image Processing (ICIP99), Kobe, Japan, 1999.

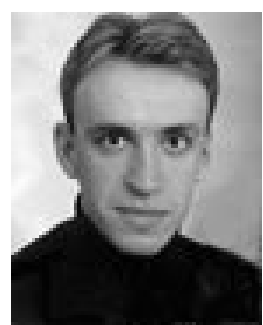

Pierre Vandergheynst (M'01) received the M.S. degree in physics and the Ph.D. degree in mathematical physics from the Université catholique de Louvain, Louvain, Belgium, in 1995 and 1998, respectively.

From 1998 to 2001, he was a Postdoctoral Researcher with the Signal Processing Laboratory, Swiss Federal Institute of Technology (EPFL), Lausanne, Switzerland. He is now Assistant Professor of visual information representation theory at EPFL where his research focuses on computer vision, image and video analysis and mathematical techniques for applications in visual information representation. He is co-Editor-in-Chief of Signal Processing.

Jean-François Gobbers, photograph and biography not available at time of publication. 